\title{
Biomarkers for Microglial Activation in Alzheimer's Disease
}

\author{
Ronald Lautner, ${ }^{1}$ Niklas Mattsson, ${ }^{1}$ Michael Schöll, ${ }^{2}$ Kristin Augutis, ${ }^{1}$ Kaj Blennow, ${ }^{1}$ \\ Bob Olsson, ${ }^{1}$ and Henrik Zetterberg ${ }^{1}$ \\ ${ }^{1}$ Clinical Neurochemistry Laboratory, Department of Neurochemistry and Psychiatry, Institute of Neuroscience and Physiology, \\ The Sahlgrenska Academy at University of Gothenburg, 43180 Mölndal, Sweden \\ ${ }^{2}$ Division of Alzheimer Neurobiology, Department of Neurobiology, Care Sciences and Society, Karolinska Institute, \\ 17177 Stockholm, Sweden
}

Correspondence should be addressed to Ronald Lautner, ronald.lautner@neuro.gu.se

Received 30 June 2011; Accepted 1 September 2011

Academic Editor: Lucilla Parnetti

Copyright (๑) 2011 Ronald Lautner et al. This is an open access article distributed under the Creative Commons Attribution License, which permits unrestricted use, distribution, and reproduction in any medium, provided the original work is properly cited.

\begin{abstract}
Intensive research over the last decades has provided increasing evidence for neuroinflammation as an integral part in the pathogenesis of neurodegenerative diseases such as Alzheimer's disease (AD). Inflammatory responses in the central nervous system (CNS) are initiated by activated microglia, representing the first line of the innate immune defence of the brain. Therefore, biochemical markers of microglial activation may help us understand the underlying mechanisms of neuroinflammation in AD as well as the double-sided qualities of microglia, namely, neuroprotection and neurotoxicity. In this paper we summarize candidate biomarkers of microglial activation in $\mathrm{AD}$ along with a survey of recent neuroimaging techniques.
\end{abstract}

\section{Introduction}

1.1. Microglia in the Healthy Brain. The central nervous system (CNS) has long been regarded as an immune privileged organ, entirely separated from the peripheral immune system. However, this concept has been fundamentally revised in recent years, as more evidence about the existence and the function of the innate immune defence of the brain has become known [1]. Microglia play an outstanding role in this context as these cells constitute the first line of defence against noxious agents in the brain. They derive from peripheral macrophages and display a ramified morphology in the resting state. It is believed that their function is to constantly scan the surrounding microenvironment in order to detect possible changes in the extracellular homeostasis of the brain that might be harmful to neurones. Upon activation, microglia proliferate migrate to the site of the lesion and undergo a drastic change in morphology, obtaining phagocytic abilities and releasing proinflammatory cytokines. The main purpose of this process is to remove cellular debris and finally to restore homeostasis in the extracellular microenvironment of the brain, thus protecting neuronal tissue from collateral damage [2].
1.2. Microglial Activation in Alzheimer's Disease-Protective or Destructive? Over the last decades, increasing evidence has suggested that neuroinflammation represents a crucial part in the pathogenesis of $\mathrm{AD}$ as well as in other neurodegenerative diseases [3]. It has been shown that activated microglia can be found in AD brains, surrounding extracellular deposits of $\beta$-amyloid, maintaining an inflammatory milieu by secreting pro-inflammatory cytokines $[4,5]$. It has been hypothesized that this chronic inflammatory state contributes decisively to the progression of the disease, thus suggesting that activated microglia not only exert neuroprotective effects, but might also be detrimental for the survival of neuronal tissue. It is still unknown why and when, in the course of $\mathrm{AD}$, microglia switch from being beneficial to becoming neurotoxic, but age-related disturbance of the physiological function and regulation of microglia (immunosenescence) has been suggested to play an important role in the $\mathrm{AD}$ pathogenesis [6]. Another question that remains to be answered is whether microglial activation occurs as a consequence of extracellular $\beta$-amyloid deposition in $\mathrm{AD}$, or if it serves as a triggering factor for $\beta$-amyloid deposition in the initial stages of the disease. In animal models, it has been shown that microglia can 
be activated by extracellular $\beta$-amyloid [7]. However, the presence of activated microglia has been observed even before the onset of $\beta$-amyloid deposition [8].

\section{Biochemical Markers}

2.1. Use of Biochemical Markers of Microglial Activation. Biochemical markers of microglial activation that allow monitoring of the inflammatory state of the CNS, might be useful for the comprehension of the double-edged characteristics of microglia in AD. Furthermore, by investigating the relation between biomarker levels and disease progression, they could contribute to the understanding of underlying pathogenic mechanisms. In addition, comparing biochemical microglial markers with neuroimaging markers will help visualize the distribution of activated microglia throughout the brain. Taking a long view, these biomarkers could also be used to monitor therapy in studies of drugs that target microglial activation.

Cerebrospinal fluid (CSF) is in direct contact with the brain parenchyma and pathological processes in the CNS often lead to altered CSF levels of specific analytes, which is the basis for using CSF biomarkers in research and clinical neurology. During the last two decades, it has become clear that several core neuropathological hallmarks of $\mathrm{AD}$ may be monitored in CSF, with low A $\beta 42$ levels secondary to amyloid pathology, and high tau and phospho-tau levels, secondary to axonal degeneration and tangle pathology, respectively [9]. CSF is also a possible source for markers of AD-related microglial activity. A number of biochemical markers of microglial activation have been described and investigated, not only in $\mathrm{AD}$, but also in other diseases which involve neuroinflammation and/or activation of peripheral cells belonging to the macrophage lineage.

2.2. Chitotriosidase. Chitotriosidase is an enzyme that exerts chitinolytic activity without having any known physiological function in humans, as inherited enzyme deficiency remains asymptomatic [10]. It appears as a product of activated mononuclear cells and functions as a marker for lipid-laden macrophages in peripheral blood, which can be used for diagnosing and therapeutic monitoring of lysosomal storage disorders such as Gaucher's disease [11]. Chitotriosidase activity in the CSF of AD patients is significantly higher than in cognitively healthy controls, which strengthens the hypothesis that disease-related microglia markers may be monitored in AD. However, CSF chitotriosidase activity appears to be unsuitable as a diagnostic tool on its own, due to large overlap between patients and controls [12].

2.3. CCL18. CCL18, also known as PARC (pulmonary activation-regulated chemokine), is a macrophage-derived chemokine that mainly attracts T-cells [13] and, precisely as chitotriosidase, shows considerably elevated levels in blood from patients with Gaucher's disease, hence serving as a suitable surrogate marker for lipid-laden macrophages in lysosomal storage disorders [14]. The presence of CCL18 in the CSF was demonstrated in patients suffering from infectious meningitis [15]. Furthermore, the expression of CCL18 in the CNS has been elucidated in relation to traumatic brain injury and cerebral tumours [16] as well as to myelinladen macrophages in and around demyelinating lesions in multiple sclerosis [17], suggesting an anti-inflammatory nature. In the previously mentioned study, CCL18 was not detected in the CSF of AD patients [12].

2.4. YKL-40. YKL-40 is a member of the same protein family as chitotriosidase (family 18 glycosyl hydrolases) and is highly similar in structure to chitotriosidase, but lacks chitinolytic activity as a result of a critical amino acid substitution. Its biological function is widely unknown, but a role in inflammation and tissue remodelling has been suggested [18]. It is released by activated macrophages and elevated concentrations have been measured in the CSF of $\mathrm{AD}$ patients [19]. However, the difference in YKL-40 between patients and controls in the study cited above was relatively small, with a large overlap between groups, and an independent smaller study could not reproduce the group difference [12], suggesting that alterations of CSF YKL-40 in AD are too small for this biomarker to be of diagnostic usability. However, more studies are needed to determine the ultimate value of YKL-40 as a biomarker for AD.

2.5. CCL2. CCL2, also known as MCP-1 (monocyte chemoattractant protein 1), is a chemokine, measurable in CSF and represents a microglial secretion factor that appears to play an important role in macrophage migration [20]. Elevated levels of CCL2 in the CSF of AD patients have been described $[21,22]$, but a recent study failed to reproduce this [12].

2.6. CD14. CD14 is a surface protein, mainly expressed by macrophages and acts as a cofactor for toll-like receptors (TLRs), which are essential for the recognition of pathogens by the innate immune system of the brain [23]. In AD, it has been shown that microglial TLRs and CD14 are involved in the inflammatory reaction surrounding $\beta$-amyloid deposits [24]. In animal models, the deletion of CD14 resulted in a change of the inflammatory response, decreasing the number of activated microglia and the amount of $\beta$ amyloid plaques [25]. One study examined cell adhesion molecules in purified monocytes from the peripheral blood of patients with $\mathrm{AD}$ and cognitively normal controls and found decreased ratios of monocytic ICAM-3/CD14 and P-selectin/CD14 in AD [26]. These results suggest that the expression of monocytic cell adhesion molecules is decreased in AD. Another interpretation is that CD14 expression per se is increased. In fact, elevated levels of a soluble form of CD14 were recently seen in CSF from $\mathrm{AD}$ and Parkinson's disease (PD) patients compared with healthy controls [27]. The lack of longitudinal data in these studies prevents from drawing any conclusion on whether upregulation of CD14 is beneficial or detrimental in the disease process. 
2.7. Neopterin. Neopterin is a degradation product deriving from the purine nucleotide guanosine triphosphate (GTP). It is secreted by macrophages upon activation and may stimulate the production of reactive oxygen species (ROS) $[28,29]$. It can be utilized as an indicator for immune system activation, showing elevated levels in several infectious diseases, autoimmune disorders, and malignant tumours as well as following allograft rejection [30]. Neopterin has even been measured in the CSF of AD patients, however without significant differences compared to controls [31].

\section{Neuroimaging}

In vivo visualization of microglial activation has become possible with the development and introduction of molecular imaging ligands (tracers) for use with positron emission tomography (PET) or single-photon emission tomography (SPECT). Most so far available imaging ligands make use of their high affinity to the peripheral benzodiazepine binding site (PBBS/PBR), also called translocator protein (TSPO), a receptor located in the outer membrane of mitochondria. Its upregulation within the CNS has been shown to reflect neuroinflammatory processes, mainly due to the activation of microglia $[32,33]$. The most extensively employed PET imaging ligand to study microglial activation in various brain diseases both in humans and transgenic animal models is the ${ }^{11}$ C-labeled isoquinoline (R)-PK11195 (1-[2-chlorophenyl]$N$-methyl- $N$-[1-methyl- propyl]-3-isoquinoline carboxamide) (PK11195), a specific ligand for the PBBS. An early PK11195 PET study that did not show any difference between $\mathrm{AD}$ patients and a group of controls suffered from several methodological issues [34]. Subsequent PET studies used the aforementioned more active R-enantiomer of PK11195 and applied different and more advanced quantification approaches to enhance tracer evaluation $[35,36]$.

One PK11195 PET study found increased binding levels in the entorhinal, temporoparietal, and cingulate cortex in a group of $\mathrm{AD}$ patients and one subject with mild cognitive impairment (MCI) as compared to normal individuals [37]. Several studies have combined PK11195 PET imaging with PET examinations using the fibrillar amyloid imaging ligand ${ }^{11} \mathrm{C}$-Pittsburgh Compound B (PIB) and dementia assessments to explore the relationship of microglial activation with underlying pathology in AD and MCI. One of these studies showed increased PK11195 binding in AD patients in comparison with normal controls in parietotemporal regions and a negative correlation with PIB retention levels in the posterior cingulate, a region that also showed lowest glucose metabolism as measured by ${ }^{18} \mathrm{~F}$-FDG PET [38]. Another study found increased PK11195 binding in frontal, temporal, parietal, occipital, and cingulate cortices and a twofold elevated PIB retention in the same cortical areas of $\mathrm{AD}$ subjects when compared to healthy controls [39]. A study in $14 \mathrm{MCI}$ patients showed that half of them had increased cortical PIB retention while five had increased PK11195 levels, and no regional correlation between the tracers was found [40]. No difference in PK11195 binding between mild and moderate AD patients, MCI patients and control subjects and no regional correlation with PIB retention were found once another study claiming that microglial activation might be associated with later stages of $\mathrm{AD}$ alone or that PK11195 might be too insensitive to detect microglial activation at the examined disease stages [41]. This is in disagreement with a study showing increases in microglial activation even during healthy aging [42].

Even if PK11195 is still considered the "gold standard", the results of PET studies in $\mathrm{AD}$ and $\mathrm{MCI}$ have been rather discordant, especially in earlier disease stages. New tracers such as $\mathrm{N}$-(2,5 dimethoxybenzyl)- $\mathrm{N}$-(4-fluoro-2phenoxyphenyl) acetamide (DAA1106) have higher binding affinity to PBBS and binding characteristics superior to PK11195. One study has so far been conducted in AD showing significantly higher binding in prefrontal, temporal, parietal, occipital, and cingulate cortices, as well as in striatum and cerebellum of $\mathrm{AD}$ patients compared with controls [43]. Another PBBS ligand, ${ }^{11} \mathrm{C}$-vinpocetine, has been suggested as potential marker for microgliosis. No difference between $\mathrm{AD}$ patients and age-matched control subjects was observed, however, disease and age-specific changes could successfully be displayed [44].

\section{Conclusions}

4.1. Clinical Applicability of Microglial Activation Markers. As microglia play a crucial role in the inflammatory response in $\mathrm{AD}$ brains, the question arises whether measuring microglial activity in the CSF or brain of AD patients might be useful in clinical routine. In order to serve as a diagnostic tool in $\mathrm{AD}$ investigations, these putative microglial biomarkers must be capable of distinguishing between $\mathrm{AD}$ patients and healthy individuals as well as from patients suffering from other types of dementia. However, current research suggests that microglial activation markers generally fail to provide high enough diagnostic accuracy to be clinically useful as diagnostic tools on their own. For comparison, the established $\mathrm{AD}$ CSF biomarker triad $(\mathrm{A} \beta 42$, totaltau and phospho-tau) has very high diagnostic accuracy when evaluated in well-controlled settings, both in crosssectional studies [45] and in longitudinal studies of early stage patients [46]. Thus, at present, microglial markers are unlikely to add diagnostic performance to the available $\mathrm{AD}$ investigation toolbox, although it cannot be excluded that they might be more useful at certain well-defined disease stages [47]. Nonetheless, biomarkers of microglial activation may give clues on underlying pathogenic mechanisms in $\mathrm{AD}$ directly in vivo in human patients, particularly concerning the ambivalence between neuroprotection and neurotoxicity following microglial activation. In addition, as microglial activation may affect the progression rate in $\mathrm{AD}$, biomarkers could be useful for monitoring of the course of the disease early on.

4.2. Further Research on Microglial Activation Markers. Future efforts investigating microglial activation markers may focus on longitudinal studies trying to elucidate whether high levels of microglial activation are beneficial or detrimental in relation to disease progression. Another 
possible aspect is to ascertain the relation between microglial biomarkers and neuroimaging markers which allow visualizing microglia in the living brain.

4.3. Possible Use of Microglial Activation Markers in Future Studies. As microglia could become a target for possible future therapies in $\mathrm{AD}$ [48], microglial activation markers may even serve as a measuring tool for evaluating and monitoring the efficiency of these therapeutic interventions. Microglial markers may also be useful to identify subgroups of patients with pronounced alteration of microglial activation, which might be most likely to respond to microglia targeting treatment.

\section{Acknowledgments}

Work in the authors' laboratory is supported by the Swedish Research Council, the Söderberg Foundation, the Lundbeck foundation, Sahlgrenska University Hospital, the Thuréus Foundation, Demensfonden, Gamla Tjänarinnor, the Göteborg Medical Society, Stiftelsen Psykiatriska Forskningsfonden, Uppsala Universitet (Medicinska Fakultetens Stiftelse för Psykiatrisk och Neurologisk Forskning), the Swedish Association of Persons with Neurological Disabilities, Alzheimer's Association, the Swedish federal government under the LUA/ALF agreement, and Swedish Brain Power.

\section{References}

[1] M. J. Carson, J. M. Doose, B. Melchior, C. D. Schmid, and C. C. Ploix, "CNS immune privilege: hiding in plain sight," Immunological Reviews, vol. 213, no. 1, pp. 48-65, 2006.

[2] J. C. M. Schlachetzki and M. Hull, "Microglial activation in Alzheimer's disease," Current Alzheimer Research, vol. 6, no. 6, pp. 554-563, 2009.

[3] M. M. Esiri, "The interplay between inflammation and neurodegeneration in CNS disease," Journal of Neuroimmunology, vol. 184, no. 1-2, pp. 4-16, 2007.

[4] J. M. Rozemuller, P. Eikelenboom, S. T. Pals, and F. C. Stam, "Microglial cells around amyloid plaques in Alzheimer's disease express leucocyte adhesion molecules of the LFA-1 family," Neuroscience Letters, vol. 101, no. 3, pp. 288-292, 1989.

[5] B. Cameron and G. E. Landreth, "Inflammation, microglia, and Alzheimer's disease," Neurobiology of Disease, vol. 37, no. 3, pp. 503-509, 2010.

[6] X. G. Luo, J. Q. Ding, and S. D. Chen, "Microglia in the aging brain: relevance to neurodegeneration," Molecular Neurodegeneration, vol. 5, no. 1, article 12, 2010.

[7] O. Wirths, H. Breyhan, A. Marcello, M. C. Cotel, W. Brück, and T. A. Bayer, "Inflammatory changes are tightly associated with neurodegeneration in the brain and spinal cord of the APP/PS1KI mouse model of Alzheimer's disease," Neurobiology of Aging, vol. 31, no. 5, pp. 747-757, 2010.

[8] M. T. Heneka, M. Sastre, L. Dumitrescu-Ozimek et al., "Focal glial activation coincides with increased BACE1 activation and precedes amyloid plaque deposition in APP[V717I] transgenic mice," Journal of Neuroinflammation, vol. 2, article 22, 2005.

[9] K. Blennow, H. Hampel, M. Weiner, and H. Zetterberg, "Cerebrospinal fluid and plasma biomarkers in Alzheimer disease," Nature Reviews Neurology, vol. 6, no. 3, pp. 131-144, 2010.

[10] R. G. Boot, G. H. Renkema, M. Verhock et al., "The human chitotriosidase gene. Nature of inherited enzyme deficiency," Journal of Biological Chemistry, vol. 273, no. 40, pp. 2568025685, 1998.

[11] J. M. Aerts, M. J. Van Breemen, A. P. Bussink et al., "Biomarkers for lysosomal storage disorders: identification and application as exemplified by chitotriosidase in Gaucher disease," Acta Paediatrica, vol. 97, no. 457, pp. 7-14, 2008.

[12] N. Mattsson, S. Tabatabaei, P. Johansson et al., "Cerebrospinal fluid microglial markers in Alzheimer's disease: elevated chitotriosidase activity but lack of diagnostic utility," Neuromolecular Medicine, vol. 13, no. 2, pp. 151-159, 2011.

[13] G. J. Adema, F. Hartgers, R. Verstraten et al., "A dendriticcell-derived C-C chemokine that preferentially attracts naive T cells," Nature, vol. 387, no. 6634, pp. 713-717, 1997.

[14] R. G. Boot, M. Verhoek, M. De Fost et al., "Marked elevation of the chemokine CCL18/PARC in Gaucher disease: a novel surrogate marker for assessing therapeutic intervention," Blood, vol. 103, no. 1, pp. 33-39, 2004.

[15] S. Kastenbauer, B. Angele, B. Sporer, H. W. Pfister, and U. Koedel, "Patterns of protein expression in infectious meningitis: a cerebrospinal fluid protein array analysis," Journal of Neuroimmunology, vol. 164, no. 1-2, pp. 134-139, 2005.

[16] C. Y. Chang, Y. H. Lee, S. J. Leu et al., "CC-chemokine ligand 18/pulmonary activation-regulated chemokine expression in the CNS with special reference to traumatic brain injuries and neoplastic disorders," Neuroscience, vol. 165, no. 4, pp. 1233$1243,2010$.

[17] L. A. Boven, M. Van Meurs, M. Van Zwam et al., "Myelinladen macrophages are anti-inflammatory, consistent with foam cells in multiple sclerosis," Brain, vol. 129, no. 2, pp. 517526, 2006.

[18] J. S. Johansen, "Studies on serum YKL-40 as a biomarker in diseases with inflammation, tissue remodelling, fibroses and cancer," Danish Medical Bulletin, vol. 53, no. 2, pp. 172-209, 2006.

[19] R. Craig-Schapiro, R. J. Perrin, C. M. Roe et al., "YKL-40: a novel prognostic fluid biomarker for preclinical Alzheimer's disease," Biological Psychiatry, vol. 68, no. 10, pp. 903-912, 2010.

[20] S. L. Deshmane, S. Kremlev, S. Amini, and B. E. Sawaya, "Monocyte chemoattractant protein-1 (MCP-1): an overview," Journal of Interferon \& Cytokine Research, vol. 29, no. 6, pp. 313-325, 2009.

[21] I. Blasko, W. Lederer, H. Oberbauer et al., "Measurement of thirteen biological markers in CSF of patients with Alzheimer's disease and other dementias," Dementia and Geriatric Cognitive Disorders, vol. 21, no. 1, pp. 9-15, 2006.

[22] D. Galimberti, N. Schoonenboom, E. Scarpini, and P. Scheltens, "Chemokines in serum and cerebrospinal fluid of Alzheimer's disease patients," Annals of Neurology, vol. 53, no. 4, pp. 547-548, 2003.

[23] T. Kielian, "Toll-like receptors in central nervous system glial inflammation and homeostasis," Journal of Neuroscience Research, vol. 83, no. 5, pp. 711-730, 2006.

[24] G. E. Landreth and E. G. Reed-Geaghan, "Toll-like receptors in Alzheimer's disease," Current Topics in Microbiology and Immunology, vol. 336, no. 1, pp. 137-153, 2009.

[25] E. G. Reed-Geaghan, Q. W. Reed, P. E. Cramer, and G. E. Landreth, "Deletion of CD14 attenuates Alzheimer's disease pathology by influencing the brain's inflammatory milieu," Journal of Neuroscience, vol. 30, no. 46, pp. 15369-15373, 2010. 
[26] T. Hochstrasser, E. Weiss, J. Marksteiner, and C. Humpel, "Soluble cell adhesion molecules in monocytes of Alzheimer's disease and mild cognitive impairment," Experimental Gerontology, vol. 45, no. 1, pp. 70-74, 2010.

[27] N. Y. Guo, H. Jeon, S. Lee, W. L. Ho, J. Y. Cho, and K. Suk, "Role of soluble CD14 in cerebrospinal fluid as a regulator of glial functions," Journal of Neuroscience Research, vol. 87, no. 11, pp. 2578-2590, 2009.

[28] C. Murr, L. C. Fuith, B. Widner, B. Wirleitner, G. BaierBitterlich, and D. Fuchs, "Increased neopterin concentrations in patients with cancer: indicator of oxidative stress?" Anticancer Research, vol. 19, no. 3 A, pp. 1721-1728, 1999.

[29] R. Sucher, K. Schroecksnadel, G. Weiss, R. Margreiter, D. Fuchs, and G. Brandacher, "Neopterin, a prognostic marker in human malignancies," Cancer Letters, vol. 287, no. 1, pp. 13-22, 2010.

[30] C. Murr, B. Widner, B. Wirleitner, and D. Fuchs, "Neopterin as a marker for immune system activation," Current Drug Metabolism, vol. 3, no. 2, pp. 175-187, 2002.

[31] S. Engelborghs, M. De Brabander, J. De Crée et al., "Unchanged levels of interleukins, neopterin, interferon$\gamma$ and tumor necrosis factor- $\alpha$ in cerebrospinal fluid of patients with dementia of the Alzheimer type," Neurochemistry International, vol. 34, no. 6, pp. 523-530, 1999.

[32] R. B. Banati, "Visualising microglial activation in vivo," GLIA, vol. 40, no. 2, pp. 206-217, 2002.

[33] S. Lang, "The role of peripheral benzodiazepine receptors (PBRs) in CNS pathophysiology," Current Medicinal Chemistry, vol. 9, no. 15, pp. 1411-1415, 2002.

[34] G. N. Groom, L. Junck, N. L. Foster, K. A. Frey, and D. E. Kuhl, "PET of peripheral benzodiazepine binding sites in the microgliosis of Alzheimer's disease," Journal of Nuclear Medicine, vol. 36, no. 12, pp. 2207-2210, 1995.

[35] A. Schuitemaker, B. N. M. van Berckel, M. A. Kropholler et al., "Evaluation of methods for generating parametric (R)[11C]PK11195 binding images," Journal of Cerebral Blood Flow and Metabolism, vol. 27, no. 9, pp. 1603-1615, 2007.

[36] G. Tomasi, P. Edison, A. Bertoldo et al., "Novel reference region model reveals increased microglial and reduced vascular binding of 11C-(R)-PK11195 in patients with Alzheimer's disease," Journal of Nuclear Medicine, vol. 49, no. 8, pp. 12491256, 2008.

[37] A. Cagnin, D. J. Brooks, A. M. Kennedy et al., "In-vivo measurement of activated microglia in dementia," The Lancet, vol. 358, no. 9280, pp. 461-467, 2001.

[38] M. Yokokura, N. Mori, S. Yagi et al., "In vivo changes in microglial activation and amyloid deposits in brain regions with hypometabolism in Alzheimer's disease," European Journal of Nuclear Medicine and Molecular Imaging, vol. 38, no. 2, pp. 343-351, 2010.

[39] P. Edison, H. A. Archer, A. Gerhard et al., "Microglia, amyloid, and cognition in Alzheimer's disease: an [11C](R)PK11195PET and [11C]PIB-PET study," Neurobiology of Disease, vol. 32, no. 3, pp. 412-419, 2008.

[40] A. Okello, P. Edison, H. A. Archer et al., "Microglial activation and amyloid deposition in mild cognitive impairment: a PET study," Neurology, vol. 72, no. 1, pp. 56-62, 2009.

[41] C. A. Wiley, B. J. Lopresti, S. Venneti et al., "Carbon 11-labeled Pittsburgh Compound B and carbon 11-labeled (R)-PK11195 positron emission tomographic imaging in Alzheimer disease," Archives of Neurology, vol. 66, no. 1, pp. 60-67, 2009.

[42] A. Schuitemaker, T. F. van der Doef, R. Boellaard et al., "Microglial activation in healthy aging," Neurobiology of Aging. In press.
[43] F. Yasuno, M. Ota, J. Kosaka et al., "Increased binding of peripheral benzodiazepine receptor in Alzheimer's disease measured by positron emission tomography with [11C]DAA1106," Biological Psychiatry, vol. 64, no. 10, pp. 835$841,2008$.

[44] B. Gulyas, A. Vas, M. Toth et al., "Age and disease related changes in the translocator protein (TSPO) system in the human brain: positron emission tomography measurements with [11C]vinpocetine," NeuroImage, vol. 56, no. 3, pp. 11111121, 2011.

[45] P. Johansson, N. Mattsson, O. Hansson et al., "Cerebrospinal fluid biomarkers for Alzheimer's disease: diagnostic performance in a homogeneous mono-center population," Journal of Alzheimer's Disease, vol. 24, no. 3, pp. 537-546, 2011.

[46] O. Hansson, H. Zetterberg, P. Buchhave, E. Londos, K. Blennow, and L. Minthon, "Association between CSF biomarkers and incipient Alzheimer's disease in patients with mild cognitive impairment: a follow-up study," The Lancet Neurology, vol. 5, no. 3, pp. 228-234, 2006.

[47] C. R. Jack Jr., D. S. Knopman, W. J. Jagust et al., "Hypothetical model of dynamic biomarkers of the Alzheimer's pathological cascade," The Lancet Neurology, vol. 9, no. 1, pp. 119-128, 2010.

[48] M. Citron, "Alzheimer's disease: strategies for disease modification," Nature Reviews Drug Discovery, vol. 9, no. 5, pp. 387$398,2010$. 


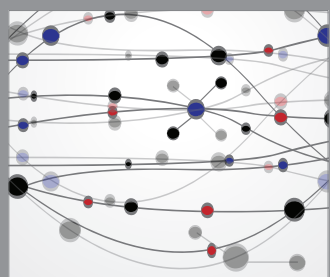

The Scientific World Journal
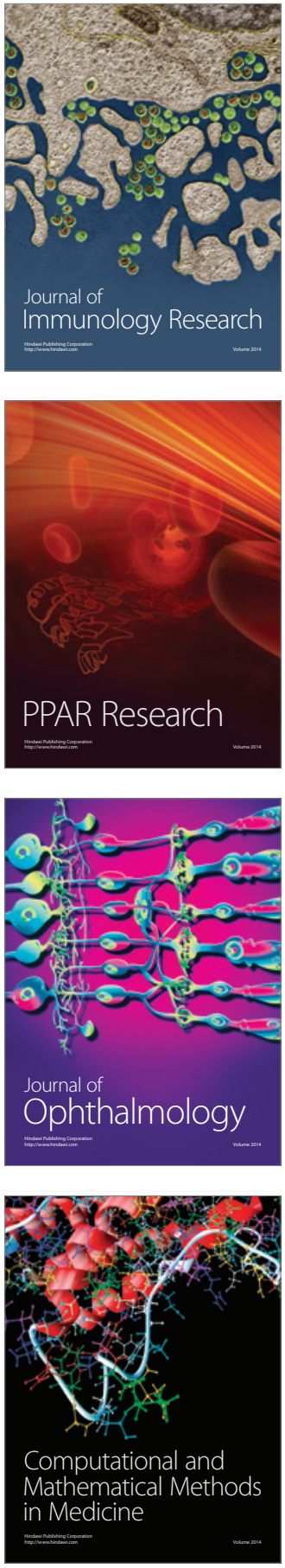

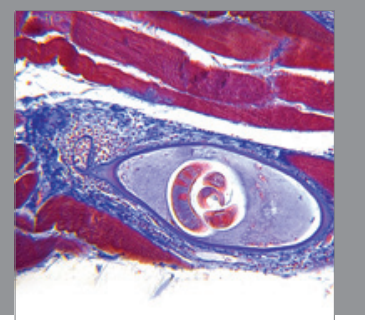

Gastroenterology

Research and Practice
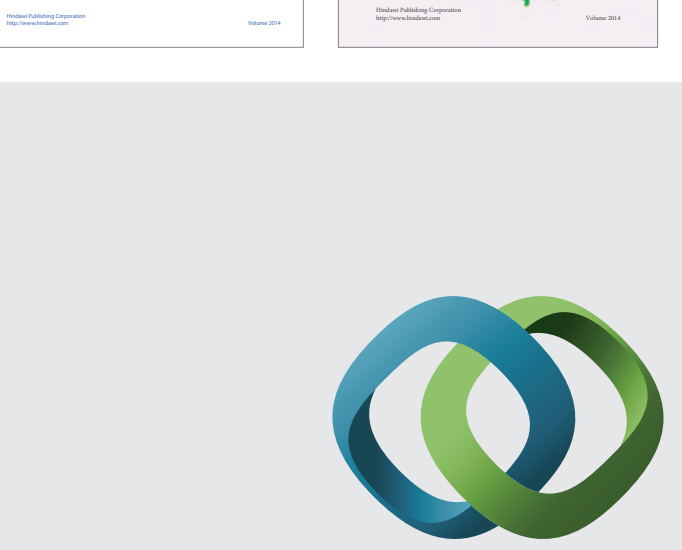

\section{Hindawi}

Submit your manuscripts at

http://www.hindawi.com
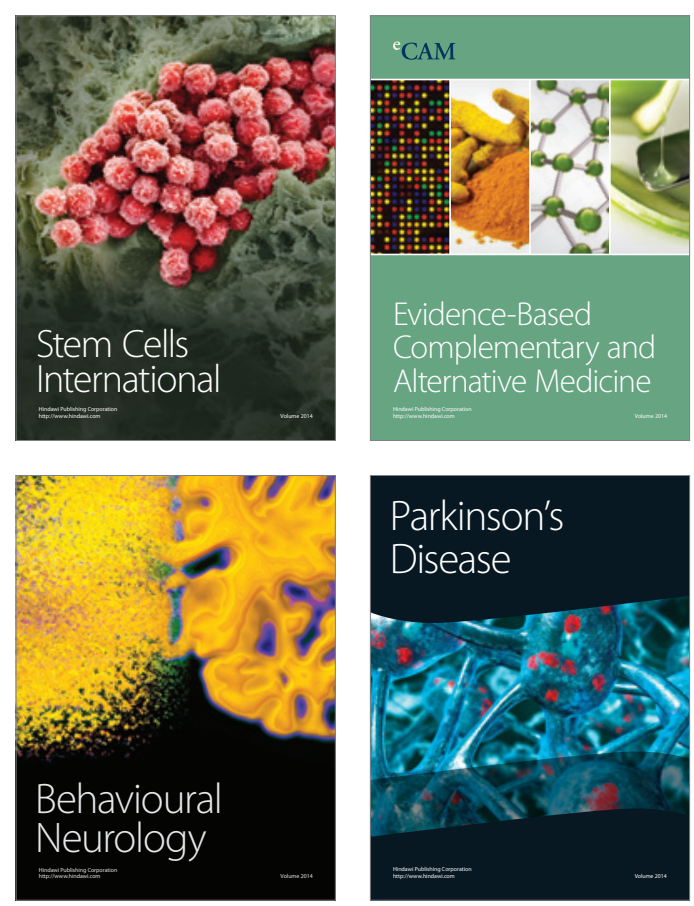

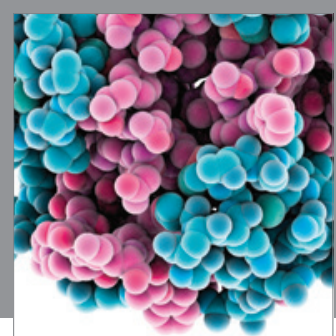

Journal of
Diabetes Research

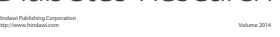

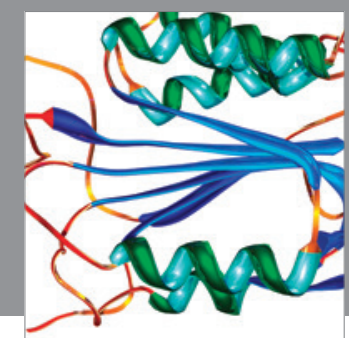

Disease Markers
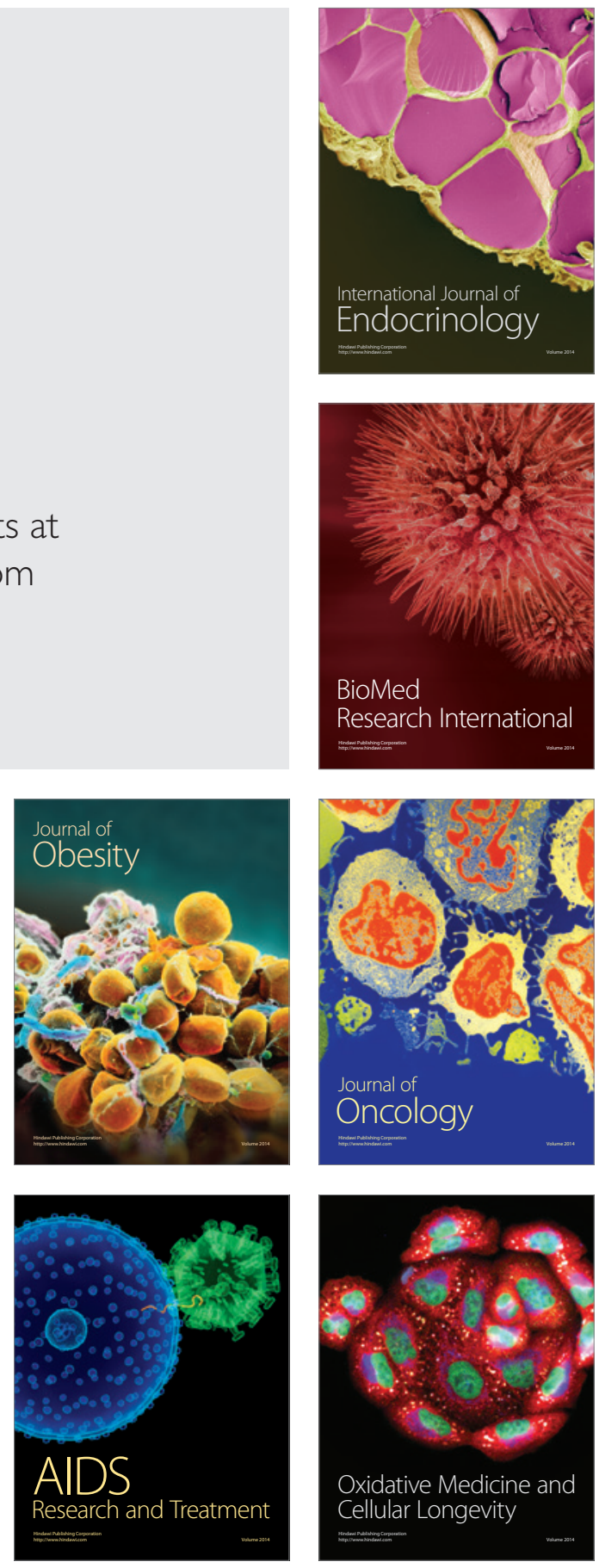